\title{
Late-onset thymidine kinase 2 deficiency: a review of 18 cases
}

\author{
Cristina Domínguez-González ${ }^{1,2,3}$, Aurelio Hernández-Laín ${ }^{4}$, Eloy Rivas ${ }^{5}$, Ana Hernández-Voth ${ }^{6}$, \\ Javier Sayas Catalán ${ }^{6}$, Roberto Fernández-Torrón ${ }^{7}$, Carmen Fuiza-Luces ${ }^{2,3,8}$, Jorge García García ${ }^{9}$, Germán Morís ${ }^{10}$, \\ Montse Olivé ${ }^{11}$, Frances Miralles ${ }^{12}$, Jordi Díaz-Manera ${ }^{3,13}$, Candela Caballero ${ }^{14}$, Bosco Méndez-Ferrer ${ }^{15}$, \\ Ramon Martîi, ${ }^{3,17}$, Elena García Arumi ${ }^{3,16}$, María Carmen Badosa ${ }^{17}$, Jesús Esteban 1,2,3, Cecilia Jimenez-Mallebrera ${ }^{3,17}$, \\ Alberto Blazquez Encinar ${ }^{2,3,8}$, Joaquín Arenas ${ }^{2,3,8}$, Michio Hirano ${ }^{18}$, Miguel Ángel Martin ${ }^{2,3,8}$ and \\ Carmen Paradas ${ }^{19,20^{*}}$
}

\begin{abstract}
Background: TK2 gene encodes for mitochondrial thymidine kinase, which phosphorylates the pyrimidine nucleosides thymidine and deoxycytidine. Recessive mutations in the TK2 gene are responsible for the 'myopathic form' of the mitochondrial depletion/multiple deletions syndrome, with a wide spectrum of severity.

Methods: We describe 18 patients with mitochondrial myopathy due to mutations in the TK2 gene with absence of clinical symptoms until the age of 12.

Results: The mean age of onset was 31 years. The first symptom was muscle limb weakness in 10/18, eyelid ptosis in $6 / 18$, and respiratory insufficiency in $2 / 18$. All patients developed variable muscle weakness during the evolution of the disease. Half of patients presented difficulty in swallowing. All patients showed evidence of respiratory muscle weakness, with need for non-invasive Mechanical Ventilation in 12/18. Four patients had deceased, all of them due to respiratory insufficiency. We identified common radiological features in muscle magnetic resonance, where the most severely affected muscles were the gluteus maximus, semitendinosus and sartorius. On muscle biopsies typical signs of mitochondrial dysfunction were associated with dystrophic changes. All mutations identified were previously reported, being the most frequent the in-frame deletion p.Lys202del. All cases showed multiple mtDNA deletions but mtDNA depletion was present only in two patients.

Conclusions: The late-onset is the less frequent form of presentation of the TK2 deficiency and its natural history is not well known. Patients with late onset TK2 deficiency have a consistent and recognizable clinical phenotype and a poor prognosis, due to the high risk of early and progressive respiratory insufficiency.
\end{abstract}

Keywords: TK2 deficiency, Mitochondrial myopathy, Multiple deletions

\section{Background}

Defects in the maintenance and repair of mitochondrial DNA (mtDNA) result in an emerging and heterogeneous group of mitochondrial disorders, caused by alterations of the nuclear genes involved in mtDNA replication [1-3].

\footnotetext{
* Correspondence: cparadas@us.es

${ }^{19}$ Neurology Department, Neuromuscular Disorders Unit, Instituto de Biomedicina de Sevilla, Hospital U. Virgen del Rocío, CSIC, Universidad de Sevilla, Avd. Manuel Siurot s/n, 41013 Sevilla, Spain

${ }^{20}$ Biomedical Network Research Centre on Neurodegenerative Diseases (CIBERNED), Madrid, Spain

Full list of author information is available at the end of the article
}

This group includes defects in enzymes involved in the maintenance of the balanced pool of deoxynucleotides of the mitochondria, which are crucial in the biosynthesis of the mitochondrial genome and have therapeutic implications $[4,5]$. The disrupted synthesis of mtDNA results in qualitative (multiple deletions) and/or quantitative (a drastic decrease in the number of copies or depletion) defects of the mtDNA. In particular, one of the 'myopathic forms' of the mitochondrial depletion/multiple deletions syndromes is caused by mutations in the TK2 gene which encodes for mitochondrial thymidine kinase, which phosphorylates the

(c) The Author(s). 2019 Open Access This article is distributed under the terms of the Creative Commons Attribution 4.0 International License (http://creativecommons.org/licenses/by/4.0/), which permits unrestricted use, distribution, and reproduction in any medium, provided you give appropriate credit to the original author(s) and the source, provide a link to the Creative Commons license, and indicate if changes were made. The Creative Commons Public Domain Dedication waiver (http://creativecommons.org/publicdomain/zero/1.0/) applies to the data made available in this article, unless otherwise stated. 
pyrimidine nucleosides thymidine ( $\mathrm{dT}$ ) and deoxycytidine (dC) $[1,6]$.

Recessive mutations in the TK2 gene (MIM\# 609560) are responsible for diverse clinical presentations mainly characterized by progressive muscle weakness, dysphagia and respiratory involvement with a wide spectrum severity and of age of onset. TK2 deficiency was initially described by Saada, et al. in 2001 [6] in four children with a severe myopathy associated with depletion of the mtDNA. Since then, a number of cases have been reported depicting a heterogeneous clinical presentation with a continuum spectrum of the disease, which includes early-onset extremely severe and rapidly progressive forms with survival of less than two years, to less severe forms with late or very late onset, and a variably slower rate of progression [7, 8]. In 2012, Tyynismaa, et al. reported the first two cases with mutations in the TK2 gene with onset in the fifth decade of life, manifesting chronic progressive external ophthalmoplegia (CPEO) associated with limb muscle weakness and dysphagia [9]. A recent publication that included 92 patients describing the natural history of this disorder proposed the classification of three clinical forms according to ages-at-onset: infantile ( $<1$ year-old), childhood (1-12) and late ( $>12$ years) onset. Nearly $40 \%$ of the reported TK2 cases presented with the symptoms prior to the age of 1 , in another $41 \%$ the onset occurred between the ages of one and 12, and only in 19\% of patients did the symptoms appeared after the age of 12 [7]. A subsequent retrospective review, with similar frequencies for those three subgroups, included eleven new cases of which only three were classified as late-onset [8]. So far, the natural history of patients with late onset TK2 deficiency has not been defined in detail.

Here, we report on the clinical features and assessments in a large series of 18 patients with late-onset TK2 deficiency, the less known and poorest defined form of this disease, to further characterize this patient subgroup. Expanding the natural history and prognosis of late-onset TK2 deficiency will facilitate earlier diagnosis and identification for treatment with therapies under clinical development.

\section{Methods}

\section{Patients}

We describe the phenotypic features of 16 Spanish and 2 US patients with mitochondrial myopathy due to mutations in the TK2 gene with the absence of clinical symptoms until the age of 12 . The series include three pairs of siblings (P3-P4, P6-P10 and P14-P15). Partial data from five patients have been previously published elsewhere (P1, P5, P9 [7], P3 and P12 [10]).

\section{Clinical evaluation}

The electronic records were reviewed to collect information about the age of onset, initial symptoms, severity, distribution and progression of the muscle weakness and extra-muscular symptoms. We gathered information from the latest neurological examination registered including, when available, the Muscle Research Council (MRC) scale to assess the muscle strength and the $6 \mathrm{~min}$ walk test (6MWT) for functional evaluation.

\section{Respiratory assessment}

The latest value of the forced vital capacity (FVC) in seated and supine position, maximum inspiratory pressure (MIP), blood gas analysis, nocturnal ventilation (assessed with nocturnal pulse oximetry and/or capnography [11] and the need for mechanical ventilation (MV) type and hours of use were recorded.

\section{Laboratory tests}

CK (creatine kinase) and lactate levels were quantified in serum in basal conditions, at diagnosis. GDF-15 (growth/differentiation factor-15) levels were quantified in plasma samples using human GDF-15 quantitative ELISA kit (R\&D Biosystems) according to the manufacturer's instructions.

\section{Muscle MRI}

Muscle MRI was performed in 8 of the 18 patients. All of them were scanned in a $1.5 \mathrm{~T}$ MR scanner (Siemens). Lower limb axial T1-weighted sequences were used for morphological analysis and short-tau inversion recovery (STIR) sequences were examined to detect muscle edema. The muscle MRI studies were evaluated by the same neurologist (R F-T) with wide experience in neuromuscular disorders. The evaluator was blind regarding the clinical manifestations. He scored pelvic, thigh and lower leg muscles in axial T1-sequences with the semiquantitative Mercuri visual scale (MVS) modified by Fisher [12]: 0: Normal appearance; 1: Mild involvement, less than $30 \%$ of individual muscle volume; 2: Moderate involvement, $30-60 \%$ of individual muscle volumes; 3 : Severe involvement, $>60 \%$ of individual muscle; 4 : End stage, all the muscle is severely affected, replaced by increased density of connective tissue and fat, with only a rim of fascia and neurovascular structures distinguishable. We compared median value of muscle fatty replacement using the Wilcoxon-Mann-Whitney test. Statistical analyses were performed using IBM SPSS Statistics, V.22 (IBM, Armonk, New York, USA).

\section{Aerobic exercise testing}

Exercise testing was performed in 5 patients on a cycle ergometer, following a ramp-like protocol (workload increases of $1 \mathrm{~W}$ every $6 \mathrm{~s}$ [averaging $10 \mathrm{~W} \cdot \mathrm{min}^{-1}$ ] starting from an initial load of $0 \mathrm{~W}$, with a pedal cadence of 60 $70 \mathrm{rpm}$ throughout the test). Gas-exchange variables were collected breath-by-breath with an automated metabolic cart (Quark CPET, COSMED, Rome, Italy). 
The peak oxygen uptake $\left(\mathrm{VO}_{2}\right.$ peak) was computed as the highest value obtained for any 10 -s period during the tests [13].

\section{Muscle biopsy}

Muscle samples were obtained by open biopsy and processed following the standard procedures: Hematoxylin and eosin (H\&E), modified Gomori trichrome, ATPase (adenosine triphosphatase), NADH (nicotinamide adenine dehydrogenase), SDH (succinate dehydrogenase), COX (cytochrome C oxidase), and COX-SDH stains were performed in all available samples. Respiratory chain enzyme activity levels were recorded when available.

\section{Genetic studies}

Molecular diagnosis was performed either by direct Sanger sequencing of exons and intron/exon boundaries of the TK2 gene, or by customized next generation sequencing (NGS) panels. Patient's skeletal muscle mtDNA deletions were investigated by long-range PCR (polymerase chain reaction) and/or Southern blot, and mtDNA copy number was assessed by quantitative PCR as previously described $[10,14]$.

The study was approved by the institutional review board of every centre and all patients signed an informed consent for the anonymous publication of this data.

\section{Results}

\section{Clinical manifestations (Table 1 )}

We included 18 patients ( 6 male, 12 female). The mean age-at-onset was 31 years (range 12 to 60 years) with a mean age at diagnosis of 48.5 years (range 23 to 73 years) resulting in an average of 17.4 years between the onset of the disease until reaching a genetic diagnosis (range 1 to 44 years). The mean duration of the disease was 19.8 years (range 6 to 44 years). Four patients from the series were deceased, all of them due to respiratory insufficiency a mean of two decades after the onset.

The first symptom was muscle limb weakness in 10/18 (55.6\%), eyelid ptosis in 6/18 (33\%) (two patients also presented ophthalmoparesis), and respiratory insufficiency in 2/18 (11.1\%). All patients developed muscle weakness during the evolution of the disease, 17/18 showing proximal and distal limb muscle weakness, 1/18 with only distal limb weakness, and 16/18 axial involvement. It is noteworthy that neck flexor weakness was clearly more severe than limb weakness (mean, 2.14 on the MRC scale).

The following muscle groups were the most frequently affected, in a symmetrical manner: shoulder abductor (mean, 4 on the MRC scale), hip flexor (mean, 3.75 on the MRC scale) and hip extensor (mean, 3.87 for both on the MRC scale) and finger extensor muscles (mean, 4.14 on the MRC scale). Four patients (22\%) lost the ability to walk without support. Facial musculature was symmetrically affected in 17 patients $(94.4 \%)$, with predominance of the orbicular oculis muscle. 16/18 of the patients (88.9\%) also had symmetrical eyelid ptosis of variable severity, with this being the first symptom in 6 patients (33.3\%). Six of them required surgical blepharoplasty due to vision impairment. Nine patients had CPEO.

The majority (11/18) had difficulty in swallowing, which resulted in severe weight loss and/or detriment to the safety of oral feeding in 6 cases, requiring percutaneous gastrostomy tube in 5 cases $(27.8 \%)$ on average 19.6 years after the onset of the disease (ranging from 12 to 28 years).

Other clinical manifestations included sensory axonal polyneuropathy $(7 / 18 ; 38.9 \%)$, neurosensory hearing loss $(3 / 18 ; 16.6 \%)$ and dysphonia due to vocal cord palsy (2/ $18 ; 11.1 \%)$. No patient had cardiomyopathy.

\section{Respiratory function}

FVC at diagnosis from the total cohort was $55.4 \%$ (ranging from 17 to 103) with a mean decrease of FVC in supine position of $8 \%$ (ranging from 0 to 14), and a mean MIP of $36.8 \%$ (ranging from 20 to $101 \%$ ), independent of the associated muscle symptoms. From a respiratory perspective, the high frequency of complications should be noted, with need for non-invasive MV in 12/18 patients (66.6\%). The mean use of the MV was $11.6 \mathrm{~h}$ per day (ranging from 8 to $24 \mathrm{~h}$ ). Eight out of the 12 patients with MV (66.6\%) presented with acute respiratory insufficiency following a routine upper respiratory infection as the first manifestation of the disease. None of these cases had any prior respiratory symptoms; however, once detected, they required MV due to hypercapnia secondary to alveolar hypoventilation. Although limb muscle weakness and/or eyelid ptosis were already present at the onset of respiratory insufficiency, those neuromuscular symptoms had not prompted a neurology consultation in any of the eight patients. Thus, the respiratory involvement resulted in the diagnosis of an underlying myopathy in these patients; the mean FVC was of $40.8 \%$ (range from 28 to 58 ) at the time of diagnosis. Of the six patients who did not needed MV, all showed evidence of respiratory muscle weakness on functional tests, although only one of them (P8) reported respiratory symptoms (orthopnoea), suggesting diaphragmatic weakness. This patient displayed ptosis and CPEO at the age of 50 associated with moderate axial and proximal limb muscle weakness ( 4 on the MRC scale). Strikingly, although the functional respiratory tests and nocturnal pulse oximetry were normal (FVC seated 103\%, FVC decubitus 100\% and MIP 101\%) nocturnal transcutaneous capnography revealed high mean levels of carbon dioxide $\left(\mathrm{CO}_{2}\right.$, mean of 48 $\mathrm{mmHg}$, with a maximum peak of $54 \mathrm{mmHg}$ ).

Four patients died of respiratory insufficiency at mean age of 56 years (ranging from 40 to 68 ), and a mean of 


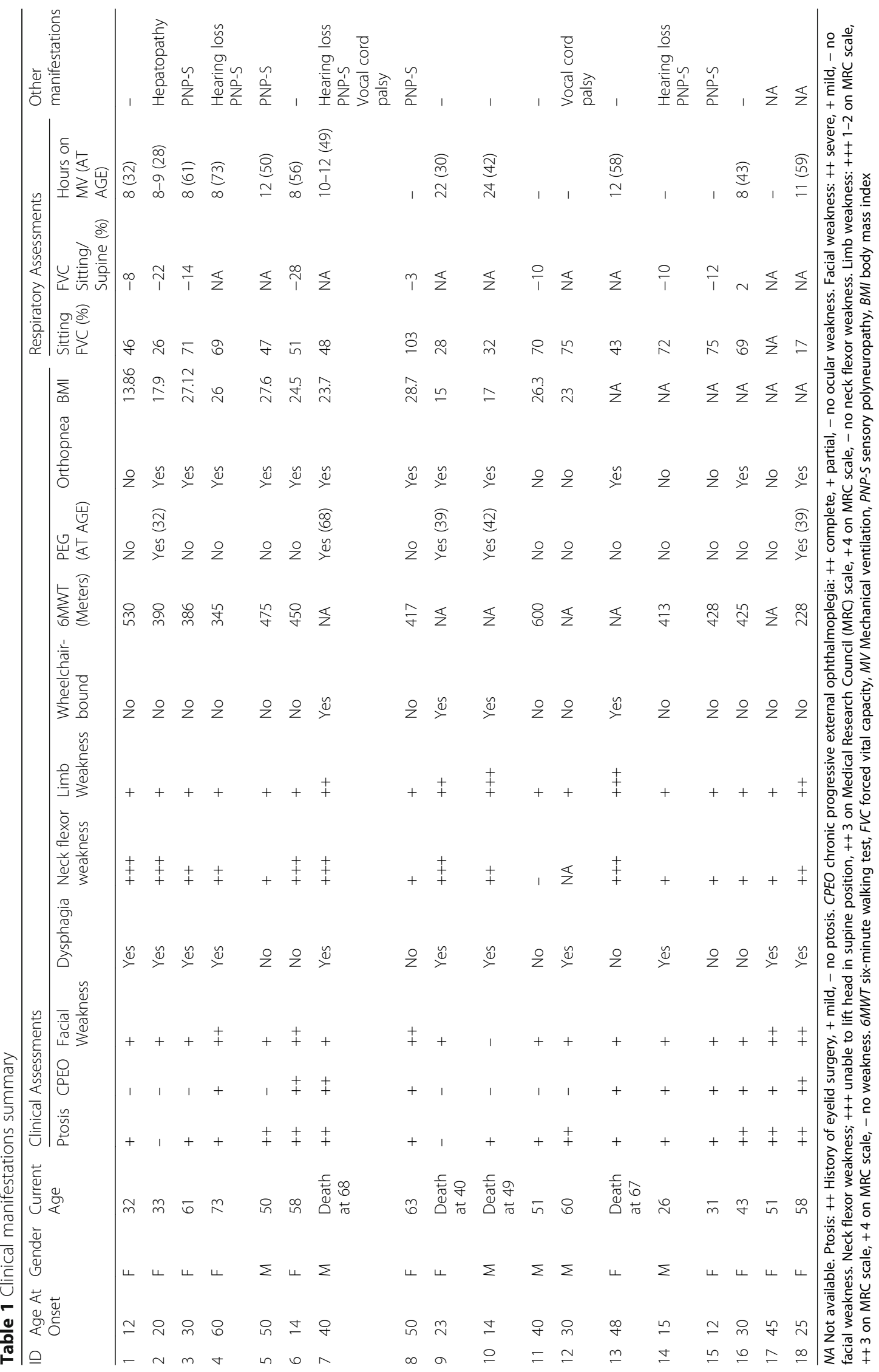


24 years after the onset of their initial symptoms (ranging from 17 to 35$)$.

\section{CK and lactate levels (Table 2)}

94.4\% of patients had increased variable serum CK levels ranging from 190 to $2435 \mathrm{UI} / \mathrm{l}$ (normal levels < $170 \mathrm{UI} /$ 1)), and $16.7 \%$ showed levels 10 -fold above the upper normal limit. Serum lactate levels were measured in basal conditions in 12 of the 18 cases. Of these, only three (25\%) displayed slightly increased levels (1.4-2x above the upper normal limit).

\section{GDF-15 levels}

GDF-15, a biomarker identified in the analysis of transcriptomic profiling of TK2 deficient human skeletal muscle [15], has been proven useful in the diagnosis of mitochondrial myopathies [16], being especially increased in patients with mitochondrial TK2 deficiency [17]. Serum levels of GDF-15 were increased in 5 out of 5 cases analysed (100\%), ranging from 1529 to $2438 \mathrm{pg} / \mathrm{mL} \quad(2113 \mathrm{pg} / \mathrm{mL} \pm 462$, mean \pm standard deviation, upper limit of normal $=550 \mathrm{pg} / \mathrm{mL}$ ) [16].

\section{Muscle MRI findings}

It was performed in 8 patients. Mean age at muscle MRI was 46.4 years old (range: $23-73$ ). Mean disease duration at the time of the scan was 18 years (range 10-31). The most severely affected muscles in axial T1-weighted sequences were the gluteus maximus, semitendinosus, sartorius and gastrocnemius medialis (median MVS: 3 ). Of these, only the gluteus maximus and sartorius were affected in all patients. Apart from the later, gluteus medius, adductor magnus and semitendinosus were also moderately affected in the thighs and gastrocnemius lateralis in the legs (median MVS: 2). No muscle fat infiltration was observed in obturator, quadratus femoris, extensoris digitorum and tibialis posterior (Fig. 1). The

Table 2 Biochemical and molecular characteristics

\begin{tabular}{|c|c|c|c|c|c|c|c|c|c|}
\hline \multirow[t]{2}{*}{$\overline{\mathrm{ID}}$} & \multicolumn{2}{|l|}{ Mutation } & \multirow{2}{*}{$\begin{array}{l}\text { Muscle } \\
\text { Biopsy }\end{array}$} & \multirow{2}{*}{$\begin{array}{l}\text { Multiple } \\
\text { Deletions }\end{array}$} & \multirow{2}{*}{$\begin{array}{l}\text { Residual } \\
\text { mtDNA (\%) }\end{array}$} & \multirow{2}{*}{$\begin{array}{l}\text { Respiratory Chain } \\
\text { Enzyme Activity }\end{array}$} & \multirow{2}{*}{$\begin{array}{l}\mathrm{CK} \\
(\mathrm{UI} / \mathrm{I})\end{array}$} & \multirow{2}{*}{$\begin{array}{l}\text { GDF-15 } \\
\text { (pg/mL) }\end{array}$} & \multirow{2}{*}{$\begin{array}{l}\text { Lactate } \\
(\mathrm{mmol} / \mathrm{l})\end{array}$} \\
\hline & Allele 1 & Allele 2 & & & & & & & \\
\hline 1 & c.323C > T (p.Thr108Met) & c.323C > T (p.Thr108Met) & Yes & Yes & 17 & $\begin{array}{l}\mathrm{Cl}, \mathrm{CII} \text { and } \mathrm{CIV} \\
\text { deficit }\end{array}$ & 2435 & 2423 & 1.95 \\
\hline 2 & c.323C > T (p.Thr108Met) & c.323C > T (p.Thr108Met) & Yes & Yes & 39 & Normal & 303 & 2439 & 2.3 \\
\hline 3 & c.604-606 AAGdel (p.Lys202del) & $\begin{array}{l}\text { c.604-606 AAGdel } \\
\text { (p.Lys202del) }\end{array}$ & Yes & Yes & 60 & CIII deficit & 294 & 1695 & 2.6 \\
\hline 4 & c.604-606 AAGdel (p.Lys202del) & $\begin{array}{l}\text { c.604-606 AAGdel } \\
\text { (p.Lys202del) }\end{array}$ & ND & ND & NA & ND & 647 & 2483 & 2.2 \\
\hline 5 & c.604-606 AAGdel (p.Lys202del) & $\begin{array}{l}\text { c.604-606 AAGdel } \\
\text { (p.Lys202del) }\end{array}$ & Yes & Yes & 66 & Normal & 357 & 1529 & 1.5 \\
\hline 6 & c.323C > T (p.Thr108Met) & c.323C > T (p.Thr108Met) & Yes & Yes & 19 & Normal & 425 & NA & 1.6 \\
\hline 7 & c.604-606 AAGdel (p.Lys202del) & $\begin{array}{l}\text { c.604-606 AAGdel } \\
\text { (p.Lys202del) }\end{array}$ & Yes & Yes & 33 & NA & 568 & NA & 2.6 \\
\hline 8 & c.604-606 AAGdel (p.Lys202del) & $\begin{array}{l}\text { c.604-606 AAGdel } \\
\text { (p.Lys202del) }\end{array}$ & Yes & Yes & NA & NA & 405 & NA & 2.4 \\
\hline 9 & c.323C > T (p.Thr108Met) & c.323C > T (p.Thr108Met) & Yes & Yes & 35 & $\begin{array}{l}\mathrm{Cl}, \mathrm{CIII} \text { and } \mathrm{CIV} \\
\text { deficit }\end{array}$ & 190 & NA & NA \\
\hline 10 & c.323C > T (p.Thr108Met) & c.323C > T (p.Thr108Met) & Yes & NA & NA & Normal & 405 & NA & 3 \\
\hline 11 & c.604-606 AAGdel (p.Lys202del) & $\begin{array}{l}\text { c.604-606 AAGdel } \\
\text { (p.Lys202del) }\end{array}$ & Yes & Yes & NA & ND & 266 & NA & NA \\
\hline 12 & c.604-606 AAGdel (p.Lys202del) & $\begin{array}{l}\text { c.604-606 AAGdel } \\
\text { (p.Lys202del) }\end{array}$ & Yes & Yes & NA & ND & 350 & NA & NA \\
\hline 13 & c.388C > T (p.Arg130Trp) & c.415G > A (p.Ala139Thr) & Yes & Yes & NA & $\begin{array}{l}\mathrm{Cl}, \mathrm{CIII} \text { and } \mathrm{CIV} \\
\text { deficit }\end{array}$ & 170 & NA & 4.14 \\
\hline 14 & c.623A > G (p.Tyr208Cys) & c.623A > G (p.Tyr208Cys) & Yes & Yes & NA & $\begin{array}{l}\mathrm{Cl}, \mathrm{CIII} \text { and } \mathrm{CIV} \\
\text { deficit }\end{array}$ & 1739 & NA & NA \\
\hline 15 & c.623A > G (p.Tyr208Cys) & c.623A > G (p.Tyr208Cys) & ND & NA & NA & ND & 381 & NA & NA \\
\hline 16 & c.323C > T (p.Thr108Met) & c.323C > T (p.Thr108Met) & Yes & Yes & 53 & Normal & 233 & NA & 1.77 \\
\hline 17 & $\begin{array}{l}\text { c.469_470insTGGG } \\
\text { (p.Asp157Valfs*11) }\end{array}$ & c. $156+6 T>G$ & Yes & Yes & 50 & NA & 537 & NA & NA \\
\hline 18 & c.604-606 AAGdel (p.Lys202del) & $\begin{array}{l}\text { c.604-606 AAGdel } \\
\text { (p.Lys202del) }\end{array}$ & NA & NA & NA & NA & 1348 & NA & NA \\
\hline
\end{tabular}



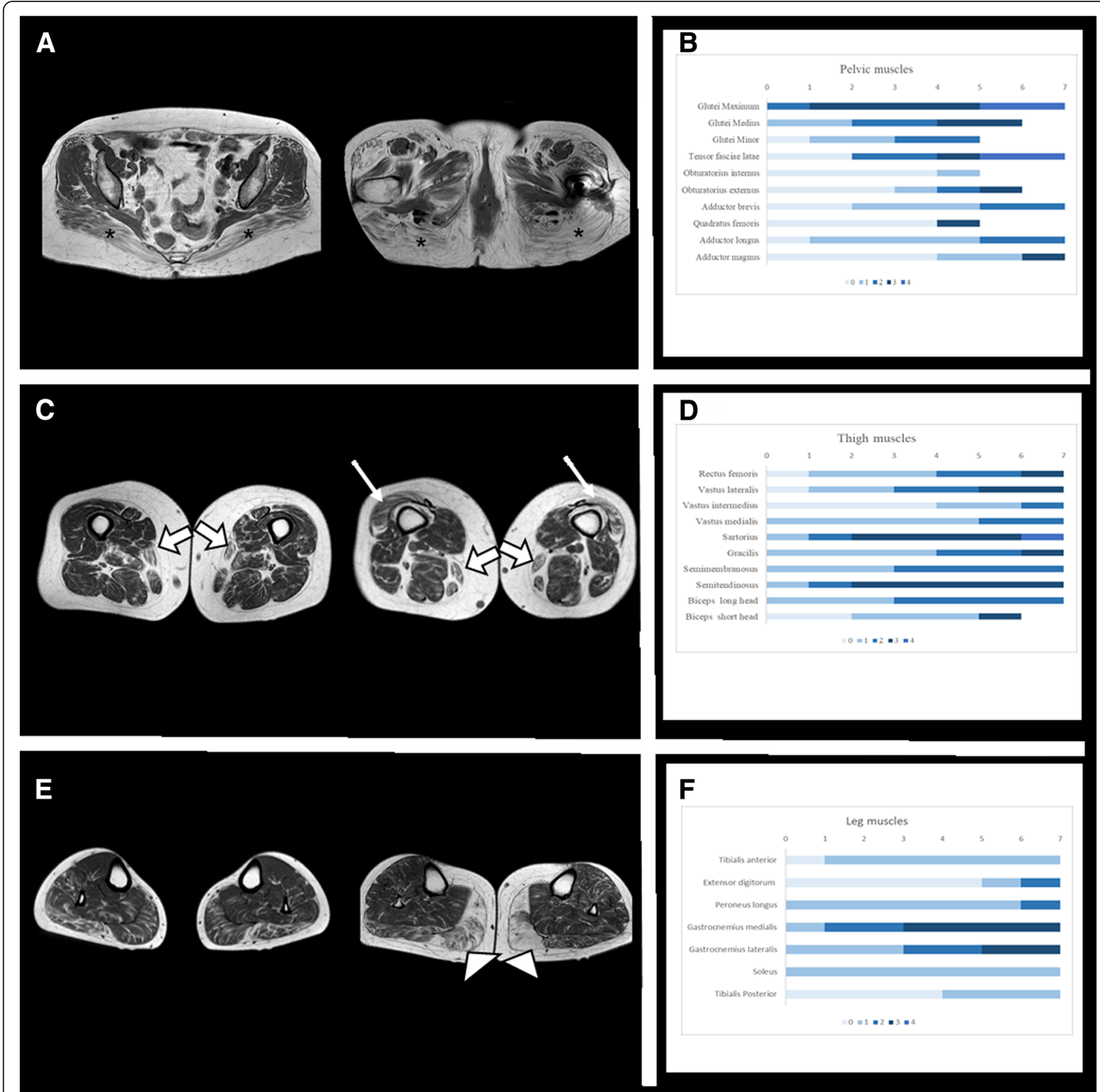

Fig. 1 Axial T1 muscle MRI and bar charts with Mercuri Visual Scale (MVS) distribution for 7 patients and per anatomical region. a, Axial T1 muscle MRI in pelvis: These two consecutive slices from different patients are showing that the gluteus maximus (marked with asterisk) is the most affected muscle. Tensor fascia latae is affected while obturator and quatratus femoris are less affected. $\mathbf{b}$, Bar chart MVs fat replacement in pelvis: MVS (0: no fat replacement, 4: the muscle is completely replaced) for all patients. Gluteus maximus is the most affected muscle, followed by tensor fascia latae. c, Axial T1 muscle MRI in thighs: These two slices from two different patients are showing the fat replacement of sartorius (wide white arrow) and vastus lateralis (thin white arrow). Other muscles like semitendinosus, semimembranosus and gracilis are also moderately affected. $\mathbf{d}$, Bar chart MVS fat replacement in thighs: MVS for all patients. Sartorius, semimembranosus, semitendinosus, gracilis and vastus lateralis are the most affected muscles. Sartorius and gracilis are affected in all patients. e, Axial T1 muscle MRI in legs: These two slices from two different patients are showing the fat replacement of gastrocnemius medialis (white arrow head). Gastrocnemius lateralis and soleus are also moderately affected. Tibialis anterior and tibialis posterior are the least affected. $\mathbf{f}$, Bar chart MVS fat replacement in legs: MVS for all patients. Gastrocnemius medialis and lateralis are the most affected muscles in legs. Tibialis anterior, extensoris digitorum and tibialis posterior are the least affected muscles 
fat replacement followed a diffuse pattern and no focal areas of fat infiltration were detected. We did not observe statistical differences regarding asymmetric involvement. STIR sequence was normal in all patients.

\section{Aerobic exercise testing}

In addition to weakness, one of the most frequent clinical manifestations in the mitochondrial myopathies is poor exercise capacity [18].The latter is reflected by low levels of $\mathrm{VO}_{2}$ peak or by poor muscle-oxygen extraction (as assessed with near-infrared spectroscopy) during graded cycle-ergometer/treadmill testing [19]. Aerobic exercise testing was performed on a cycle ergometer in five patients. The mean $\pm \mathrm{SD} \mathrm{VO}_{2}$ peak obtained was $14.8 \pm 3.2 \mathrm{~mL} / \mathrm{kg}^{-1} / \mathrm{min}^{-1}$, with normal consumption values of $40.0 \pm 9.5 \mathrm{~mL} / \mathrm{kg}^{-1} / \mathrm{min}^{-1}$ [20].

\section{Muscle biopsies}

Muscle biopsies were performed in 16 patients, 11 were available to re-analysis. The morphological study revealed numerous ragged-red fibers in $100 \%$ of the biopsies, which were hyper-reactive with SDH reaction and usually COX-deficient. COX-deficient fibers accounted for approximately $5-15 \%$ of all fibers. Frequently these muscles also showed dystrophic features with frequent necrotic fibers, some with phagocytosis, and increased endomysial connective tissue (present in 7 out of 11 biopsies revised). Marked type I fiber predominance was also observed in 2 patients (Fig. 2). These findings differ from the usual pattern displayed in other mitochondrial myopathies, where the typical signs of mitochondrial proliferation and dysfunction are not associated with other relevant changes in muscle histology structure [21]. We have results of the analysis of the enzymatic activity of respiratory chain complexes of 10 patients. Only in half of them a reduction in the activity of one or more enzymatic complexes were identified (Table 2).

\section{Genetic studies}

All patients harbored biallelic mutations in the $T K 2$ gene (Ref.Seq. NM_004614.4) (Table 2). Most patients $(16 / 18 ; 88.9 \%)$ were homozygous. All mutations were previously reported $[7,8]$, with the in-frame deletion p.Lys202del (c.604_606AAGdel) being the most frequent (16/36 alleles; $44.4 \%)$, followed by the missense mutation p.Thr108Met (c.323C > T) $\quad(12 / 36 ; 27.8 \%)$. Additionally, three missense mutations were identified in 3 patients: p.Arg130Trp (c.388C > T), p.Ala139Thr (c.415G > A), and p.Tyr208Cys (c.623A > G). Finally, one patient harbored a frameshift mutation p.Asp157 Valfs*11(c.469-470insTGGG) in compound heterozygosis with a splice site mutation c. $156+6 \mathrm{~T}>\mathrm{G}$. Genetic data from patients P1, P2, P5, P9 and P12, were previously reported $[7,10]$. Muscle mtDNA copy-number was studied in 9 patients and severe mtDNA depletion was detected in only two (17\% of residual mtDNA in $\mathrm{P} 1$ and $19 \%$ of residual mtDNA in P6). Fourteen out of 14 patients (100\%) showed the presence of multiple mtDNA deletions in muscle.

\section{Discussion}

The late-onset presentation of TK2 deficiency is the least frequent clinical mode of presentation known. These patients are considered to have a milder presentation than those with infancy and childhood onset disease, however, few cases have been described to date and those reported were not extensively explored. So far, 17 patients with late-onset were reported to harbour TK2 biallelic mutations [7-10, 22]. However clinical details were scarce, heterogeneous, and reports did not clearly define the phenotype or rate of progression of the disease. In some cases, clinical presentation is similar to that described in the childhood onset patients, with progressive limb, facial, extraocular, oropharyngeal and respiratory muscle weakness, but with a slower progression, whereas in other cases, CPEO is the main manifestation [9]. Respiratory insufficiency has been mentioned as a potential cause of death although comprehensive data about the respiratory involvement is not available for all the previously published patients: severe respiratory insufficiency is described in $41 \%$ of the reported cases but in the remaining $59 \%$ this data is unavailable or superficially described [7, 8, 22].

We identified 16 Spanish and two North American patients, from 13 different families, with TK2 mutations and a late-onset presentation. Exhaustive clinical description is here provided to facilitate earlier and accurate diagnosis and to improve the knowledge of the natural history of this rare, and probably underdiagnosed disorder.

The clinical features and results of the diagnostic tests described in our series show a homogeneous phenotypic pattern in late-onset TK2 deficiency consisting of progressive proximal limb, axial neck flexor and facial muscle weakness frequently associated with ptosis, ophthalmoparesis and bulbar weakness, along with an early and severe, although unrecognized, respiratory involvement. Diaphragmatic weakness is very characteristic, occurring in all of our cases, showing an early onset but slow progression; 12/18 (66.6\%) required MV during the evolution of the disease and in $8 / 18$ (44.4\%) was the cause for the first medical consultation. This pattern of respiratory involvement was found even in patients who only had an apparently isolated CPEO phenotype. Therefore, it is critical to identify signs of nocturnal hypoventilation during clinical evaluation of these patients, regardless of the severity of the skeletal myopathy. This discrepancy between diaphragmatic and limb weakness was also reflected in some patients with virtually 


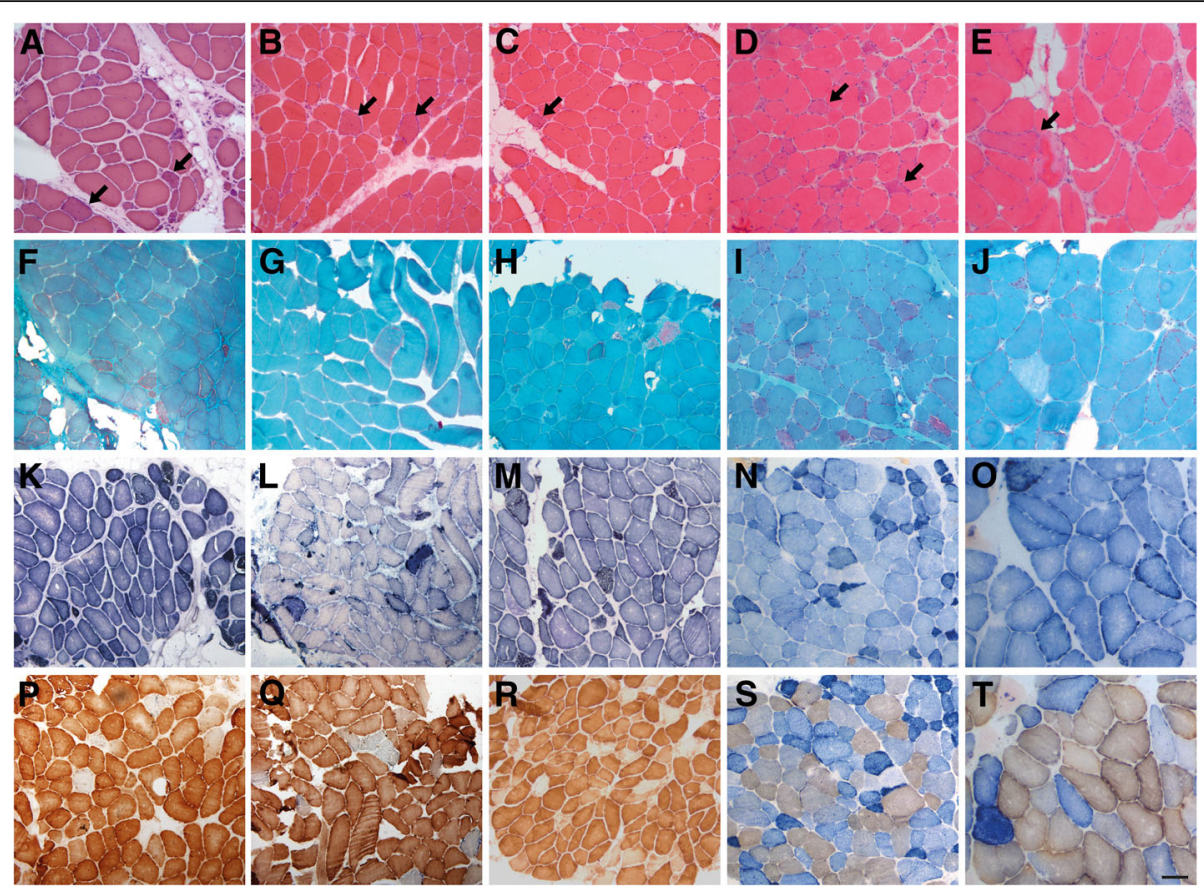

Fig. 2 Morphological alterations in muscle biopsies from patients P1 (a, f, $\mathbf{k}, \mathbf{p})$, P5 (b, g, l, q), P9 (c, h, m, r), P14 (D, i, n, s) and P16 (e, j, o, t). a-e H\&E shows dystrophic features in all cases with mild endomysal fibrosis, adipose tissue replacement, atrophy and necrotic fibers. Ragged-red fibers are frequently identified in all muscle samples (arrows). $\mathbf{f}$-j Gomori trichrome showed the characteristic ragged-red fibers in all the biopsies. k-o Succinate dehydrogenase (SDH) reveals an increase of the oxidative staining in numerous fibers. $\mathbf{p}$-t Frequent cytochrome $\mathbf{C}$ oxidase (COX) deficient fibers are present in variable proportion in the different cases ( $\mathbf{p}$ and $\mathbf{r}$, COX staining; $\mathbf{o}, \mathbf{s}$ and $\mathbf{t}$, COX-SDH combined staining). Scale bar $=100 \mu \mathrm{m}$

normal 6MWT results, despite using MV (see Table 1). In our series, the capnography was the most sensitive test for detecting the respiratory dysfunction, since it was abnormal even before basal FVC and MIP revealed alterations.

Muscle biopsies showed the typical findings of mitochondrial dysfunction described in most mitochondrial myopathies. However, as in other TK2 deficiency forms, they also revealed dystrophic features which are distinct from the majority of other mitochondrial myopathies. Thus, our data support that the association of both mitochondrial and dystrophic pattern strongly suggest mutations in the TK2 gene as the underlying cause.

All previously published late-onset patients showed multiple mtDNA deletions, while mtDNA depletion was found only in one of the five cases in whom the mtDNA copy-number was quantified. Our findings corroborate the previous results indicating the presence of multiple mtDNA deletions is more frequent than mtDNA depletion in the late-onset TK2 deficient patients. Previous reports showed that mtDNA depletion is found in the most of early onset patients [7], but our data support that it cannot be considered a valid prognostic marker since it can also be found in late-onset cases.

In muscle MRI the fat muscle replacement was diffuse, resembling many muscular dystrophies and congenital myopathies. Muscle degeneration in MRI was described in five MERRF patients with the m.8344A > G mutation [23], and more recently fatty infiltration has been communicated in patients with single, large-scale deletions of mitochondrial DNA [24]. However, no extensive studies have been published trying to define muscle MRI patterns in different mitochondrial myopathies. So, there is no specific MRI pattern for any mitochondrial myopathy described so far. In our series of TK2 patients although no clear pattern of fat infiltration was detected, we have identified some radiological common features, as the involvement of the sartorius muscle in all cases. This muscle is usually spared until late stages in many genetic muscle diseases (is only affected early in some myofibrillar myopathies, in the Laing distal myopathy and in RYR1-related myopathies (encodes for ryanodine receptor 1 protein) [12, 25-27]), so this finding could be helpful for differential diagnosis.

Serum GDF-15 levels have recently been revealed as a sensitive and specific biomarker for the diagnosis of mitochondrial myopathies [16, 17]. In our series, it proved to be very high in all analysed cases, so it could orientate the molecular diagnosis in a proper clinical context, before the muscle biopsy was performed.

As in other mitochondrial myopathies [19], in our series the cardiopulmonary exercise testing identified a 
very reduced consumption of oxygen, even in patients with CPEO as a predominant clinical manifestation (P8). This indicates that, although the weakness may not be severe in late-onset TK2 deficiency patients, the exercise capacity is abnormally low, ultimately impairing physical activity.

Noticeably, the p.Lys202del was the most frequent mutation in the TK2 gene in our series of late-onset patients, which is consistent with the finding that this mutation appears to be restricted to adult-onset cases, since it has not been reported in any infantile-onset patients who have not even harbouring this mutation in a single allele [8]. Nevertheless, it was reported in one patient with childhood-onset, who was compound heterozygous for this mutation and a frameshift mutation, and began showing symptoms at 2.5 years but survived until 8.5 years-old [28]. The eight cases with this mutation in our series were all homozygous supporting the idea that this mutation is associated with a milder effect (age at onset ranging from 25 to 60 years). Interestingly, this mutation has only been identified in 13 unrelated Spanish patients ((11, 13, 26, 27, and this study), 2 related patients from Hispanic ethnic background [10], and one patient from Venezuela (this study) suggesting that it could be a private mutation and that Spanish/Hispanic candidate patients may be amenable for a rapid genetic screening of this mutation. However, haplotype analysis would be required to confirm the possible founder effect of this mutation. The p.Thr108Met mutation was the second most common mutation in this study, however it has been found in infantile and childhood onset cases $[6,7]$ of different geographic origin.

TK2 deficiency is a severe disorder causing premature death. In recent pre-clinical studies, it has been demonstrated that treatment with pyrimidine nucleosides $(\mathrm{dC}$ $+\mathrm{dT}$ ) in the H126N knock-in mouse model of TK2 deficiency, leads to a prolonged life span in the animals and a restored mtDNA copy number, without significant toxicity [4]. This opens the door to a potential therapeutic intervention in humans with this metabolic hereditary disorder, making it necessary to define sensitive and objective outcomes to assess an eventual response to treatment. Our findings suggest that functional respiratory tests, serum GDF-15 level and the stress cyclometer evaluation are potentially good candidates for monitoring the progression of disease.

\section{Conclusion}

In summary, our study shows that late-onset patients with mitochondrial TK2 deficiency have a consistent and recognizable clinical phenotype, characterized by a progressive myopathy with predominant facial and axial neck flexor weakness, and respiratory involvement, often associated to CPEO. Their prognosis is poor, due to the high risk of early and progressive respiratory insufficiency. Yet, some patients may present with a severe acute respiratory failure. Early detection of respiratory involvement requires an active search in the clinics, even in asymptomatic patients. A small number of rationally designed treatments are being developed for mitochondrial disorders [29], including nucleoside substrate enhancement therapy designed specifically for TK2 deficiency [4]. Therefore, early diagnosis of TK2 deficiency is important as patients could benefit from the existence of a potential therapy.

\begin{abstract}
Abbreviations
6MWT: 6-min walking test; ATPase: Adenosine triphosphatase; BMI: Body mass index; $\mathrm{CK}$ : Creatine kinase; $\mathrm{CO}_{2}$ : Carbon dioxide; $\mathrm{COX}$ : Cytochrome $\mathrm{C}$ oxidase; CPEO: Chronic progressive external ophthalmoplegia; dC: Deoxycytidine; dT: Thymidine; FVC: Forced vital capacity; GDF-15: Growth differentiation factor 15; H\&E: Hematoxylin and eosin; MIP: Maximal inspiratory pressure; MRC: Muscle Research Council; MRI: Magnetic resonance imaging; mtDNA: Mitochondrial DNA; MV: Mechanical ventilation; MVS: Mercury visual scale; NADH: Nicotinamide adenine dehydrogenase; NGS: Next generation sequencing; PCR: Polymerase chain reaction; SDH: Succinate dehydrogenase; STIR: Shor tau inversion recovery; $\mathrm{VO}_{2}$ peak: Peak oxygen uptake
\end{abstract}

\section{Acknowledgements}

Not applicable.

\section{Funding}

This work was supported by research grants of Plan Nacional de I + D + I and Instituto de Salud Carlos III (ISCIII), Subdirección General de Evaluación y Fomento de la Investigación Sanitaria", project PI16-01843 (CP), PI16/00579 and CP09/00011 for CJM and the European Regional Development Fund (FEDER a way to achieve Europe). MAM has received funding from the Spanish ISCIII (grant PI 15/00431). A multicentric grant funded by the ISCIII (PMP15/00025 to MAM, RM, MO, CP).

\section{Availability of data and materials}

The datasets used and/or analysed during the current study are available from the corresponding author on reasonable request.

\section{Authors' contributions}

CDG, AHV, JSC, JG, GM, MO, FM, JDM, CC, JBM, JE, MH and CP handled patients and recollected clinical data for the manuscript. RT collected and analysed the MRI data of the patients. EG and ABE performed molecular analysis; CB and CJ provided GDF-15 analysis. AH and ER collected and review muscle biopsy data. CD and CP coordinated all the study. CD, MAM and CP wrote the initial manuscript. RM, JA, MAM, CP, and MH provided critical discussion of the research. All authors contributed to the final version of the manuscript. All authors read and approved the final manuscript.

\section{Ethics approval and consent to participate}

Written informed consent was obtained from patients for publication of anonymised clinical data.

\section{Consent for publication}

Not applicable.

\section{Competing interests}

$\mathrm{MH}$ and RM are co-inventors on patent applications filed by Columbia University Medical Center (CUMC) for deoxynucleoside therapy for mitochondrial DNA depletion syndromes including TK2 deficiency. The patent applications and other intellectual property have been licensed by CUMC to Meves Pharmaceuticals, Inc. CUMC may be eligible to receive payments related to the development and commercialization of the technology. Any potential licensing fees earned will be paid to CUMC and are shared with inventors through CUMC distribution policy. MH and RM are paid consultants to Meves Pharmaceutical, Inc. The rest of authors declare that they have no conflict of interest. 


\section{Publisher's Note}

Springer Nature remains neutral with regard to jurisdictional claims in published maps and institutional affiliations.

\begin{abstract}
Author details
${ }^{1}$ Neurology department, Neuromuscular disorders Unit, 12 de Octubre Hospital, Madrid, Spain. ${ }^{2}$ Research Institute i+12, 12 de Octubre Hospital, Madrid, Spain. ${ }^{3}$ Biomedical Network Research Centre on Rare Diseases (CIBERER), Instituto de Salud Carlos III, Madrid, Spain. ${ }^{4}$ Neuropathology, Pathology Department, Neuromuscular disorders Unit, 12 de Octubre Hospital, Madrid, Spain. ${ }^{5}$ Pathological Anatomic Department, Neuromuscular Disorders Unit, Instituto de Biomedicina de Sevilla, Hospital U. Virgen del Rocío, CSIC, Universidad de Sevilla, Sevilla, Spain. ${ }^{6}$ Neumology department, Neuromuscular disorders Unit, 12 de Octubre Hospital, Madrid, Spain. ${ }^{7}$ Neurology Department, Neuromuscular disorders Unit, Hospital de Donostia, San Sebastian, Spain. ${ }^{8}$ Mitochondrial and Neuromuscular Diseases Laboratory, Research Institute of Hospital '12 de Octubre' ('i+12'), Madrid, Spain. ${ }^{9}$ Neurology Department, Hospital de Albacete, Albacete, Spain. ${ }^{10}$ Neurology Department, Neuromuscular disorders Unit, Hospital Central de Asturias, Oviedo, Spain. ${ }^{11}$ Pathological Anatomy Department, Neuromuscular disorders unit, IDIBELL-Hospital de Bellvitge, Barcelona, Spain. ${ }^{12}$ Neurology department, Neuromuscular disorders unit, Hospital Universitari Son Espases, Palma, Spain. ${ }^{13}$ Neurology department, Neuromuscular disorders unit, Hospital de la Santa Creu I Sant Pau, Barcelona, Spain. ${ }^{14}$ Respiratory Department, Instituto de Biomedicina de Sevilla, Hospital U. Virgen del Rocío, CSIC, CIBERES, Universidad de Sevilla, Sevilla, Spain. ${ }^{15}$ Rehabilitation Department, Hospital Virgen del Rocio, Sevilla, Spain. ${ }^{16}$ Research group on Neuromuscular and Mitochondrial Diseases, Valld'Hebron Research Institute, Universitat Autònoma de Barcelona, Barcelona, Spain. ${ }^{17}$ Neuromuscular Unit, Neurology Department, Institut de Recerca Sant Joan de Déu, Hospital Sant Joan de Déu, Barcelona, Spain. ${ }^{18}$ Department of Neurology, H. Houston Merritt Center, Columbia University Medical Center, New York, New York, USA.

${ }^{19}$ Neurology Department, Neuromuscular Disorders Unit, Instituto de Biomedicina de Sevilla, Hospital U. Virgen del Rocío, CSIC, Universidad de Sevilla, Avd. Manuel Siurot s/n, 41013 Sevilla, Spain. ${ }^{20}$ Biomedical Network Research Centre on Neurodegenerative Diseases (CIBERNED), Madrid, Spain.
\end{abstract}

Received: 5 February 2019 Accepted: 17 April 2019

Published online: 06 May 2019

\section{References}

1. Viscomi C, Zeviani M. MtDNA-maintenance defects: syndromes and genes. J Inherit Metab Dis. 2017;40(4):587-99.

2. El-Hattab AW, Craigen WJ, Scaglia F. Mitochondrial DNA maintenance defects. Biochim Biophys Acta. 2017;1863(6):1539-55

3. El-Hattab AW, Scaglia F. Mitochondrial DNA depletion syndromes: review and updates of genetic basis, manifestations, and therapeutic options. Neurotherapeutics. 2013;10(2):186-98.

4. Lopez-Gomez C, Levy RJ, Sanchez-Quintero MJ, Juanola-Falgarona M, Barca E, Garcia-Diaz B, et al. Deoxycytidine and Deoxythymidine treatment for thymidine kinase 2 deficiency. Ann Neurol. 2017;81(5):641-52.

5. Cámara Y, González-Vioque E, Scarpelli M, Torres-Torronteras J, Caballero A Hirano M, et al. Administration of deoxyribonucleosides or inhibition of their catabolism as a pharmacological approach for mitochondrial DNA depletion syndrome. Hum Mol Genet. 2014;23(9):2459-67.

6. Saada A, Shaag A, Mandel H, Nevo Y, Eriksson S, Elpeleg O. Mutant mitochondrial thymidine kinase in mitochondrial DNA depletion myopathy. Nat Genet. 2001;29(3):342-4.

7. Garone C, Taylor RW, Nascimento A, Poulton J, Fratter C, DomínguezGonzález C, et al. Retrospective natural history of thymidine kinase 2 deficiency. J Med Genet. 2018;55:515-21.

8. Wang J, Kim E, Dai H, Stefans V, Vogel H, Al Jasmi F, et al. Clinical and molecular spectrum of thymidine kinase 2-related mtDNA maintenance defect. Mol Genet Metab. 2018:124:124-30.

9. Tyynismaa H, Sun R, Ahola-Erkkilä S, Almusa H, Pöyhönen R, Korpela M, et al. Thymidine kinase 2 mutations in autosomal recessive progressive external ophthalmoplegia with multiple mitochondrial DNA deletions. Hum Mol Genet. 2012;21(1):66-75.

10. Cámara Y, Carreño-Gago L, Martín MA, Melià MJ, Blázquez A, Delmiro A, et al. Severe TK2 enzyme activity deficiency in patients with mild forms of myopathy. Neurology. 2015;84(22):2286-8.
11. Trucco F, Pedemonte M, Fiorillo C, Tan HL, Carlucci A, Brisca G, et al Detection of early nocturnal hypoventilation in neuromuscular disorders. Int Med Res. 2018;46(3):1153-61.

12. Fischer D, Kley RA, Strach K, Meyer C, Sommer T, Eger K, et al. Distinct muscle imaging patterns in myofibrillar myopathies. Neurology. 2008;71(10): 758-65.

13. Fiuza-Luces C, Díez-Bermejo J, Fernández-de la Torre M, Rodríguez-Romo G, Sanz-Ayán P, Delmiro A, et al. Health benefits of an innovative exercise program for mitochondrial disorders. Med Sci Sports Exerc. 2018;50:1142-51.

14. Andreu AL, Martinez R, Marti R, García-Arumí E. Quantification of mitochondrial DNA copy number: pre-analytical factors. Mitochondrion. 2009;9(4):242-6

15. Kalko SG, Paco S, Jou C, Rodríguez MA, Meznaric M, Rogac M, et al. Transcriptomic profiling of TK2 deficient human skeletal muscle suggests a role for the p53 signalling pathway and identifies growth and differentiation factor-15 as a potential novel biomarker for mitochondrial myopathies. BMC Genomics. 2014;15:91.

16. Montero R, Yubero D, Villarroya J, Henares D, Jou C, Rodríguez MA, et al. GDF-15 is elevated in children with mitochondrial diseases and is induced by mitochondrial dysfunction. PLoS One. 2016;11(2):e0148709.

17. Yatsuga S, Fujita Y, Ishii A, Fukumoto Y, Arahata H, Kakuma T, et al. Growth differentiation factor 15 as a useful biomarker for mitochondrial disorders. Ann Neurol. 2015;78(5):814-23.

18. Taivassalo T, Jensen TD, Kennaway N, DiMauro S, Vissing J, Haller RG. The spectrum of exercise tolerance in mitochondrial myopathies: a study of 40 patients. Brain. 2003;126(Pt 2):413-23.

19. Fernández J, Montemayor T, Bautista J, Márquez R, Jiménez L, Arenas J, et al. The use of cardiopulmonary exercise test in patients with mitochondrial myopathies. Med Clin (Barc). 2000;114(4):121-7.

20. Aspenes ST, Nilsen TI, Skaug EA, Bertheussen GF, Ellingsen $\varnothing$, Vatten $L$, et al. Peak oxygen uptake and cardiovascular risk factors in 4631 healthy women and men. Med Sci Sports Exerc. 2011;43(8):1465-73.

21. Milone M, Wong LJ. Diagnosis of mitochondrial myopathies. Mol Genet Metab. 2013;110(1-2):35-41.

22. Alston CL, Schaefer AM, Raman P, Solaroli N, Krishnan KJ, Blakely EL, et al. Late-onset respiratory failure due to TK2 mutations causing multiple mtDNA deletions. Neurology. 2013:81(23):2051-3.

23. Catteruccia M, Sauchelli D, Della Marca G, Primiano G, Cuccagna C, Bernardo D, et al. "Myo-cardiomyopathy" is commonly associated with the A8344G "MERRF" mutation. J Neurol. 2015;262(3):701-10.

24. Hedermann G, Dahlqvist JR, Løkken N, Vissing CR, Knak KL, Andersen LK, et al. Progressive fat replacement of muscle contributes to the disease mechanism of patients with single, large-scale deletions of mitochondrial DNA. Neuromuscul Disord. 2018:28(5):408-13.

25. Carbonell-Corvillo P, Tristán-Clavijo E, Cabrera-Serrano M, Servián-Morilla E, García-Martín G, Villarreal-Pérez L, et al. A novel MYH7 founder mutation causing Laing distal myopathy in Southern Spain. Neuromuscul Disord. 2018;28:828-36

26. Olivé M, Odgerel Z, Martínez A, Poza JJ, Bragado FG, Zabalza RJ, et al. Clinical and myopathological evaluation of early- and late-onset subtypes of myofibrillar myopathy. Neuromuscul Disord. 2011;21(8):533-42.

27. Tordjman M, Dabaj I, Laforet P, Felter A, Ferreiro A, Biyoukar M, et al. Muscular MRI-based algorithm to differentiate inherited myopathies presenting with spinal rigidity. Eur Radiol. 2018;28:5293-303.

28. Martí R, Nascimento A, Colomer J, Lara MC, López-Gallardo E, Ruiz-Pesini E, et al. Hearing loss in a patient with the myopathic form of mitochondrial DNA depletion syndrome and a novel mutation in the TK2 gene. Pediatr Res. 2010;68(2):151-4.

29. Hirano M, Emmanuele V, Quinzii CM. Emerging therapies for mitochondrial diseases. Essays Biochem. 2018;62(3):467-81. 